\title{
Computed Tomography and Clinical Features Differentiating Coronavirus Disease 2019 from Seasonal Influenza Pneumonia
}

\section{Shuang Zhao}

Sichuan University West China Hospital

\section{Zixing Huang}

Sichuan University West China Hospital

Hanjiang Zeng

Sichuan University West China Hospital

\section{Zhixia Chen}

Sichuan University West China Hospital

\section{Fengming Luo}

Sichuan University West China Hospital

\section{Chongwei Zhang}

Sichuan University West China Hospital

Bin Song ( $\square$ songlab_radiology@163.com )

Sichuan University West China Hospital

\section{Research Article}

Keywords: Infection; Coronavirus; Influenza, Human; Pneumonia, Viral; Tomography, X-ray computed

Posted Date: May 26th, 2020

DOl: https://doi.org/10.21203/rs.3.rs-31186/v1

License: (c) (1) This work is licensed under a Creative Commons Attribution 4.0 International License. Read Full License 


\section{Abstract}

Objectives: To investigate computed tomography (CT) and clinical features could help differentiate coronavirus disease 2019 (COVID-19) from seasonal influenza pneumonia.

Methods: We retrospectively evaluated the clinical features and chest CT findings of Chinese patients with COVID-19 and seasonal influenza pneumonia treated during the same period.

Results: The 24 patients with COVID-19 (mean age, 41 years; 13 men) and 79 patients with seasonal influenza pneumonia (mean age, 41 years; 50 men) differed significantly in mean temperature, respiratory rate, and systolic blood pressure; in central-peripheral, superior-inferior, and anterior-posterior distribution but not lateral distribution of pulmonary lesions; and patchy ground-glass opacity (GGO), GGO nodules, vascular enlargement in GGO, air bronchogram, bronchiolectasis in GGO or consolidation, interlobular septal thickening, and crazy-paving pattern. Separate regression models were developed with clinical features, CT features (including anatomical distributions), and a combined model informed by the first two. The combined model had the best diagnostic performance for identifying COVID-19: a cut-off value of 0.38 was $74 \%$ sensitive and $100 \%$ specific and had an area under the receiver operating characteristics curve of 0.94 . This model was based on sputum production, vascular enlargement in GGO, and centralperipheral distribution (random vs subpleural).

Conclusions: The combination of sputum production, vascular enlargement in GGO, and centralperipheral distribution should be extremely helpful in the differential diagnosis of COVID-19.

\section{Introduction}

A new coronavirus, severe acute respiratory syndrome coronavirus 2 (SARS-CoV-2), emerged in Wuhan, China, at the end of 2019. The World Health Organization (WHO) named the disease caused by this pathogen coronavirus disease 2019 (COVID-19). After COVID-19 spread to more than 100 countries and led to hundreds of thousands of cases within few months.

Influenza is a mild-to-severe, contagious respiratory illness caused by the influenza viruses. Serious outcomes include hospitalization or death, and the elderly, the young, and people with certain health conditions are at a high risk of these complications. The most common form of influenza is seasonal influenza. The two main types of seasonal influenza virus, $A$ and $B$, are routinely spread among humans and are responsible for seasonal influenza epidemics each year. Influenza accounts for thousands of deaths and hospital admissions annually in the USA, with an even greater impact in developing countries $^{1-4}$.

COVID-19 and seasonal influenza pneumonia are similar in terms of transmission and symptoms. In both cases, the primary modes of transmission are droplets and contact transmission. Fever, chills, headache, myalgia, malaise, and anorexia accompanied by respiratory symptoms, including nonproductive cough and sore throat, are the main symptoms of both ${ }^{5-7}$. Reverse transcription polymerase chain reaction (RT- 
PCR) is a highly recommended diagnostic technique. However, some studies have indicated that PCR results are susceptible to false negatives from several factors ${ }^{8-10}$.

CT has become the indispensable means not only in the early detection but also in monitoring the clinical course, evaluating the disease severity, and may be presented as an important warning signal preceding the negative RT-PCR test results ${ }^{11}$. Almost all patients suspected of having the virus should undergo chest CT. However, we found no CT studies describing differences between the emerging viral pneumonia caused by COVID-19 and influenza pneumonia, the most common viral pneumonia. We sought to determine whether initial chest CT findings and clinical features could differentiate between COVID-19 and seasonal influenza pneumonia.

\section{Materials And Methods}

\section{Patients and chest CT}

This retrospective study was approved and the requirement for informed consent was waived by the Biomedical Research Ethics Committee of West China Hospital of Sichuan University. The study followed the principles of the Declaration of Helsinki with voluntary participation. The data were analyzed and handled in an anonymous format. We adhered to relevant guidelines and regulations in all experiments.

We enrolled patients with RT-PCR-confirmed COVID-19 presenting between January 21, 2020, and February 9, 2020 at a single tertiary referral hospital in Sichuan province, China. Patients co-infected with other pathogens, in severe respiratory distress, or who required oxygen at any time during the disease course were excluded. Patients in whom COVID-19 was suspected or confirmed underwent CT with a Revolution, GE Healthcare CT scanner (Milwaukee, Wisconsin). The CT protocol was: tube voltage, $120 \mathrm{kV}$ (automatic adjustment); tube current, 200 500mAs; rotation time, 0.5 second; section thickness, 0.625 $\mathrm{mm}$; collimation, $0.625 \mathrm{~mm}$; pitch, 1 ; matrix, $512 \times 512$; and inspiration breath hold. Reconstruction was performed with a bone algorithm with a thickness of $1 \mathrm{~mm}$ and an interval of $1 \mathrm{~mm}$.

Patients with influenza seen during the same period were included if they were admitted to our hospital, tested positive for influenza A or B viruses, and had thin-section CT scans available for review. (These scans were obtained with a Somatom Definition AS+ CT scanner; Siemens Healthcare, Forchheim, Germany). The CT protocol was: tube voltage, 120 kV; tube current, $110 \mathrm{mAs}$ (automatic adjustment); rotation time, 0.5 second; section thickness, $0.75 \mathrm{~mm}$; collimation, $0.6 \mathrm{~mm}$; pitch, 1 ; matrix, $512 \times 512$; and inspiration breath hold. Reconstruction was performed with a bone algorithm with a thickness of $1 \mathrm{~mm}$ and an interval of $1 \mathrm{~mm}^{12}$.

All scans were obtained with the patient in the supine position during end-inspiration without intravenous contrast material. The following windows were used: a mediastinal window with a window width of 350 $\mathrm{HU}$ and a window level of $40 \mathrm{HU}$ and a lung window with a width of $1800 \mathrm{HU}$ and a level of $-400 \mathrm{HU}^{12}$. 
Clinical information, collected from the medical record, included age, sex, symptoms, and travel and exposure history.

\section{CT Image Review}

All CT images were reviewed by two fellowship-trained cardiothoracic radiologists with approximately 10 years of experience each. Images were reviewed independently, and final decisions were reached by consensus. Disagreements were resolved by a third fellowship-trained cardiothoracic radiologist with 15 years of experience.

The distribution of lung abnormalities was recorded as: (a) Left, right or bilateral lung; (b) Predominantly subpleural (involving the peripheral 1/3 of the lung), hilar (involving around the hilum), and random ( without predilection for subpleural or around hilum); (c) Predominantly superior ( superior to the bifurcation of trachea), inferior (inferior to the bifurcation of trachea), and random ( without predilection for superior or inferior); (d) Predominantly anterior ( anterior to the horizontal line across the axillary midline), posterior ( posterior to the horizontal line across the axillary midline), and random ( without predilection for anterior or posterior) ; (e) Presented as a solitary lesion. Numbers of involved lobes and segments of lungs were recorded also.

The CT findings included ground-glass opacity (GGO), consolidation, crazy-paving pattern, bronchiolectasis, interlobular septal thickening, and lymphadenopathy. Other abnormalities were noted; for example, cavitation, air bronchogram, reticulation, calcification, subpleural curvilinear line, halo sign, pleural effusion, pleural thickening, and pericardial effusion.

\section{Statistical analysis}

Independent Student's t-tests were used to compare two groups of normally distributed variables, and the Mann-Whitney $\mathrm{U}$ test was used for two groups of nonnormally distributed variables. For $2 \times 2$ contingency tables, Fisher's exact test was used when one or more cells had an expected count less than 1 ; continuity correction was used when all expected counts were greater than 1 but at least one cell was less than 5; otherwise, the chi-square test was used. For contingency tables, Fisher's exact test was used when more than $20 \%$ of the cells were less than 5 ; otherwise, the chi-square test was used.

Variables for which more than $30 \%$ of values were missing were not included in logistic regression analysis. Considering the small sample size, the variables were divided into two groups: clinical features and CT features, including anatomical distribution. In each group, individual variables were included in an unadjusted analysis, and those significantly associated with the presence of pneumonia were considered for inclusion in multiple logistic regression analyses to identify independent predictors of pneumonia for each disease. Then, the independent predictors in each of the two groups were combined in a third multiple logistic regression analysis using backward stepwise selection, based on the Akaike information criterion, to identify the independent predictors of each disease from all variables. 
For all logistic regression analyses, odds ratios and 95\% confidence intervals of independent predictors were calculated; model function equations, sensitivity, specificity, and area under the curve (AUC) were given.

All statistical analyses were performed with SPSS 20.0 (IBM, Armonk, NY, USA). A two-tailed p value lower than 0.05 was considered statistically significant.

\section{Results}

\section{Features and clinical manifestations}

We enrolled 24 patients with COVID-19 and 79 with influenza. Temperature and respiratory rate were significantly higher in patients with influenza than in patients with COVID-19, whereas systolic pressure was lower in patients with influenza (Table 1). The proportion of patients who had cough, sputum production, and sore throat was significantly higher in the influenza group than in the COVID-19 group. 
Table 1. Characteristics of 103 Patients with Influenza or COVID-19 Infections

Anatomic distribution of lung lesions and imaging findings

The CT findings were negative in 15 of 79 patients with influenza and were positive in all 24 patients with COVID-19. Overall, lung lesions in patients with COVID-19 were most likely to appear in the subpleural, inferior, and posterior lung fields, whereas those in patients with influenza are mostly randomly distributed, without obvious regional distribution characteristics (Table 2). Patients with COVID-19 were more likely to have isolated lesions than were patients with influenza.

\begin{tabular}{|c|c|c|c|}
\hline Characteristic & $\begin{array}{l}\text { Influenza } \\
(n=79)\end{array}$ & $\begin{array}{l}\text { COVID-19 } \\
(n=24)\end{array}$ & $p$ \\
\hline Sex, m/f & $50 / 29$ & $13 / 11$ & 0.48 \\
\hline Age mean (SD), years & $40.9(19.5)$ & $46.17(17.4)$ & 0.12 \\
\hline Smokers, n (\%) & $12(15.19)$ & $2(8.33)$ & 0.49 \\
\hline Alcoholics, n (\%) & $13(16.46)$ & $2(8.33)$ & 0.50 \\
\hline Days from illness onset mean (SD) & $7.04(8.85)$ & $5.91(6.79)$ & 0.84 \\
\hline Temperature, mean (SD), ${ }^{\circ} \mathrm{C}$ & $38.14(1.12)$ & $37.61(0 v 86)$ & $0.04 *$ \\
\hline Respiratory rate, mean (SD), bpm & $20.70(2.07)$ & $19.92(1.50)$ & $0.02 *$ \\
\hline Systolic pressure, mean (SD), mm Hg & $128.58(15.81)$ & $137.71(14.26)$ & $0.03 *$ \\
\hline Diastolic pressure, mean (SD), $\mathrm{mmHg}$ & $80.29(14.38)$ & $85.17(14.76)$ & 0.08 \\
\hline Oxygen saturation, n (\%) & $96.02(3.39)$ & $96.79(1.38)$ & 0.78 \\
\hline \multicolumn{4}{|l|}{ Symptom, n (\%) } \\
\hline Fever & $61(77.22)$ & $17(70.83)$ & 0.666 \\
\hline Rigor & $8(10.13)$ & 0 & 0.246 \\
\hline Chills & $16(20.25)$ & $7(29.17)$ & 0.319 \\
\hline Cough & $63(79.75)$ & $11(45.83)$ & 0.002 \\
\hline Sputum production & $44(55.70)$ & $2(8.33)$ & $<0.001$ \\
\hline Chest tightness & 9 (11.39) & $2(8.33)$ & 0.997 \\
\hline Dyspnea & $14(17.72)$ & $1(4.17)$ & 0.201 \\
\hline Sore throat & $17(21.52)$ & 0 & 0.033 \\
\hline Fatigue & $18(22.78)$ & $5(20.83)$ & 0.893 \\
\hline Headache & $14(17.72)$ & $1(4.17)$ & 0.201 \\
\hline Myalgia & $19(24.05)$ & $4(16.67)$ & 0.484 \\
\hline Abdominal pain & $2(2.53)$ & $2(8.33)$ & 0.222 \\
\hline Diarrhea & $2(2.53)$ & $2(8.33)$ & 0.222 \\
\hline
\end{tabular}

Data are expressed as mean \pm standard deviation or number of cases (percentage).

${ }^{*} p \square 0.05$ 
Table 2. Computed Tomography Findings in 103 Patients with Influenza or COVID-19 Infections

\begin{tabular}{|c|c|c|c|}
\hline Finding & $\begin{array}{l}\text { Influenza } \\
(n=64)\end{array}$ & $\begin{array}{l}\text { COVID-19 } \\
(n=24)\end{array}$ & $P$ \\
\hline Laterality distribution, (\%) & & & 0.08 \\
\hline Left lobe & $7(10.94)$ & $3(12.50)$ & \\
\hline Right lobe & $8(12.50)$ & $3(12.50)$ & \\
\hline Bilateral lobe & $49(76.56)$ & $18(75.00)$ & \\
\hline Central-peripheral distribution, (\%) & & & $<0.001$ \\
\hline Hilar & 0 & $1(4.17)$ & \\
\hline Subpleural & $23(35.94)$ & $19(79.67)$ & \\
\hline Random & $41(64.06)$ & $4(16.67)$ & \\
\hline Superior-inferior distribution, (\%) & & & 0.005 \\
\hline Superior & $5(7.81)$ & 0 & \\
\hline Inferior & $27(42.19)$ & $17(70.83)$ & \\
\hline Radom & $32(50.00)$ & $7(29.17)$ & \\
\hline Anterior-posterior distribution, (\%) & & & 0.01 \\
\hline anterior & $2(3.13)$ & $1(4.17)$ & \\
\hline posterior & $18(28.13)$ & $12(50.00)$ & \\
\hline random & $44(68.76)$ & $11(45.83)$ & \\
\hline Solitary lesion, (\%) & $2(3.13)$ & $5(20.83)$ & 0.02 \\
\hline Number of involved lobes & $2.68(1.93)$ & $3.54(1.67)$ & 0.052 \\
\hline Number of involved segments & $6.57(6.62)$ & $8.04(5.82)$ & 0.20 \\
\hline Patchy GGO, n (\%) & $36(56.25)$ & $20(83.33)$ & 0.001 \\
\hline GGO nodule, $\mathrm{n}(\%)$ & $13(20.31)$ & $9(37.5)$ & 0.03 \\
\hline $\mathrm{Q} 3 \mathrm{~cm}$ round like GGO, $\mathrm{n}(\%)$ & 0 & $1(4.17)$ & 0.23 \\
\hline Vascular enlargement in GGO, $\mathrm{n}(\%)$ & $7(10.94)$ & $16(66.67)$ & $<0.001$ \\
\hline Consolidation, n (\%) & $25(39.06)$ & $4(16.67)$ & 0.15 \\
\hline Air bronchogram, n (\%) & $20(31.25)$ & $15(62.50)$ & 0.001 \\
\hline Bronchiolectasis within lesion, $\mathrm{n}(\%)$ & $15(23.44)$ & $14(58.33)$ & $<0.001$ \\
\hline Interlobular septal thickening, $\mathrm{n}$ (\%) & & & 0.004 \\
\hline Outside GGO & $8(12.50)$ & $2(8.33)$ & \\
\hline Within GGO & $7(10.94)$ & $9(37.50)$ & \\
\hline Crazy-paving pattern, n (\%) & $1(1.56)$ & $3(12.50)$ & 0.039 \\
\hline Solid nodule, n (\%) & $35(54.69)$ & $11(45.83)$ & 0.90 \\
\hline Halo sign nodule, n (\%) & $3(4.69)$ & $2(8.33)$ & 0.72 \\
\hline Reticular pattern, n (\%) & $6(9.38)$ & $3(12.50)$ & 0.74 \\
\hline Subpleural curvilinear line, $\mathbf{n}(\%)$ & $7(10.94)$ & $1(4.17)$ & 0.75 \\
\hline Linear opacity, n (\%) & $42(65.63)$ & $11(45.83)$ & 0.53 \\
\hline Cavity, n (\%) & $1(1.56)$ & 0 & 0.99 \\
\hline Pleural thickening, n (\%) & $15(23.44)$ & $2(8.33)$ & 0.34 \\
\hline Pleural effusion, $\mathrm{n}$ (\%) & $12(18.75)$ & 0 & 0.10 \\
\hline Lymphadenopathy, n (\%) & $10(15.63)$ & $2(8.33)$ & 0.83 \\
\hline Atelectasis, n (\%) & $2(3.13)$ & 0 & 0.99 \\
\hline Emphysema, n (\%) & $7(10.94)$ & $1(4.17)$ & 0.75 \\
\hline
\end{tabular}


Abbreviation: GGO, ground-glass opacity

The following CT features were more prevalent in patients with COVID-19 than in patients with influenza (Fig. 1-3): patchy GGO, GGO nodule, vascular enlargement, air bronchogram, bronchiolectasis, and crazypaving pattern (Table 2). Interlobular septal thickening in patients with COVID-19 was more likely to appear within the GGO lesions, whereas in patients with influenza, it was more likely to appear outside the GGO lesions.

\section{Differentiating COVID-19 from influenza pneumonia}

We used single-factor logistic regression analysis to screen for predictors across the clinical features and CT features, including anatomic distributions (Tables 3 and 4). Clinical features, including temperature, systolic pressure, cough, and sputum production were included in the first preliminary logistic regression analysis. The logistic regression formula of clinical feature factors was as follows: Logit (Clinical features $)=-8.985+0.044 \times$ Systolic pressure $+2.763 \times$ Sputum $($ no $=1 /$ yes $=0)$. The cut-off value, sensitivity, specificity and AUC of Logit(no-CT) was $0.295,0.783,0.747$ and 0.819 , respectively. The second preliminary logistic regression analysis was based on CT features and the anatomic distribution of lesions. The logistic regression formula of anatomic distribution and CT features factors was as follows: Logit $($ CT features $)=-4.802+1.984 \times$ GGO nodule $(y e s=1 /$ no $=0)+3.440 \times$ vascular enlargement in GGO(yes $=1 /$ no $=0)+3.332 \times$ distribution(random $=1 /$ subpleural $=0)$. The cut-off value, sensitivity, specificity and AUC of Logit (CT features) was $0.415,0.750,0.953$ and 0.889 , respectively. Finally, a logistic regression model combined the variables from the clinical features and CT features models. The combined model contained three features: sputum production ( $b, 3.688)$, vascular enlargement in the GGO (b, 3.955), and central-peripheral distribution (vs random distribution; $b, 3.711)$. The logistic regression formula of both Clinical features and CT features factors was as follows: Logit $($ Combined $)=-8.138+3.688 \times \operatorname{Sputum}($ no $=1 /$ yes $=0)+3.955 \times$ vascular enlargement in GGO(yes $=1 / \mathrm{no}=0)+3.711 \times$ Central-peripheral distribution $($ random $=1 /$ subpleural $=0)$. The cut-off value, sensitivity, specificity and AUC of Logit (Combined) was $0.38,0.74,1.000$ and 0.94 , respectively. The ROC curves of Logit (Clinical features), Logit (CT features) and Logit (Combined) are shown in Fig. 4. 
Table 3. Performance Characteristics of Three Simple Logistic Regression Models in the Diagnosis of COVID-19 Infection

\begin{tabular}{|c|c|c|c|}
\hline & Clinical features $^{\mathrm{a}}$ & $\begin{array}{l}\text { Anatomic distribution and CT } \\
\text { features }^{\mathrm{b}}\end{array}$ & Both Clinical and CT features ${ }^{\mathrm{C}}$ \\
\hline $\begin{array}{l}\text { Performance } \\
\text { characteristic }\end{array}$ & $\begin{array}{l}\text { Logit }(\text { no-CT) }= \\
-8.985+0.044 \times \\
\text { systolic blood } \\
\text { pressure }+2.763 \times \\
\text { sputum production } \\
(\text { no }=1 / \text { yes }=0)\end{array}$ & $\begin{array}{l}\text { Logit }(C T)=-4.802+1.984 \times \text { GGO } \\
\text { nodule }(\text { yes }=1 / \text { no }=0)+3.440 \times \\
\text { vascular enlargement in GGO (yes } \\
=1 / \text { no }=0)+3.332 \times \text { distribution } \\
(\text { random }=1 / \text { subpleural }=0 \text { ) }\end{array}$ & $\begin{array}{l}\text { Logit }(\text { Merge })=-8.138+3.688 \times \\
\text { sputum }(\text { no }=1 / \text { yes }=0)+3.955 \times \\
\text { vascular enlargement in GGO (yes }= \\
1 / \text { no }=0)+3.711 \times \text { central-peripheral } \\
\text { distribution (random }=1 / \text { subpleural }= \\
\text { 0) }\end{array}$ \\
\hline $\mathrm{R}^{2}$ & 0.33 & 0.61 & 0.76 \\
\hline Cutoff & 0.30 & 0.42 & 0.38 \\
\hline Sensitivity, \% & 78 & 75 & 74 \\
\hline Specificity, \% & 75 & 95 & 100 \\
\hline AUC & 0.82 & 0.89 & 0.94 \\
\hline
\end{tabular}

Abbreviation: AUC, area under the receiver operating characteristics curve

${ }^{a}$ Including temperature, systolic pressure, cough, and sputum production

${ }^{\mathrm{b}}$ Solitary lesion, random distribution in the central-peripheral distribution category, inferior distribution in the superior-inferior distribution category, patchy GGO, GGO nodule, vascular enlargement in GGO, air bronchogram, bronchiolectasis within lesions, and crazy-paving pattern

c Systolic pressure, sputum, GGO nodule, vascular enlargement in GGO, and random distribution in the centralperipheral distribution 
Table 4. Unadjusted Analysis of Associations Between Individual Variables and COVID-19 or Influenza Pneumonia.

\begin{tabular}{|c|c|c|}
\hline Variables & $P$ & OR $(95 \% \mathrm{CI})$ \\
\hline \multicolumn{3}{|l|}{ Clinical features } \\
\hline Temperature & 0.04 & 0.63 (0.41 to 0.98$)$ \\
\hline Systolic pressure & 0.03 & 1.03 (1.00 to 1.06$)$ \\
\hline Cough & 0.003 & $4.582(1.697$ to 12.370$)$ \\
\hline Sputum production & 0.001 & 13.588 (2.978 to 61.997$)$ \\
\hline \multicolumn{3}{|l|}{ Anatomical distribution } \\
\hline Solitary lesion & 0.01 & $0.117(0.021$ to 0.651$)$ \\
\hline \multicolumn{3}{|l|}{ Central-peripheral distribution } \\
\hline Subpleural (reference) & 0.002 & \\
\hline Random & $<0.001$ & $0.118(0.036$ to 0.389$)$ \\
\hline \multicolumn{3}{|l|}{ Superior-inferior distribution } \\
\hline Random(reference) & 0.13 & \\
\hline Inferior & 0.04 & $2.878(1.040$ to 7.969$)$ \\
\hline \multicolumn{3}{|l|}{ CT features } \\
\hline GGO patchy & 0.003 & $5.972(1.870$ to 19.075$)$ \\
\hline GGO nodule & 0.03 & $3.046(1.100$ to 8.432$)$ \\
\hline Vascular enlargement in GGO & $<0.001$ & 20.571 (6.514 to 64.966$)$ \\
\hline Air bronchogram & 0.001 & 4.917 (1.865 to 12.965$)$ \\
\hline Bronchiectasis within focus & $<0.001$ & $5.973(2.226$ to 16.031$)$ \\
\hline Crazy-paving pattern & 0.04 & $11.143(1.102$ to 112.696$)$ \\
\hline
\end{tabular}

Abbreviation: GGO, ground-glass opacity

\section{Discussion}

As a newly emerging respiratory virus infection, a novel coronavirus, COVID-19 is highly infectious ${ }^{13}$. The present COVID-19 outbreak has occurred in the influenza season ${ }^{3}$. he two infections have much in common, including being caused by similar viruses and producing similar symptoms, especially pneumonia, making the differential diagnosis difficult in most clinical settings. Given the newness of COVID-19 however, studies comparing it to the flu have not been done. Thus, we aimed to determine whether the two infections could be differentiated with clinical features, CT features, or some combination of the two.

We found that patients with COVID-19 had fevers that were on average $0.53^{\circ} \mathrm{C}$ lower than those in patients with influenza pneumonia $\left(37.61^{\circ} \mathrm{C}\right.$ vs. $38.14^{\circ} \mathrm{C}$, respectively) and were more likely to have a dry cough with less sputum production than were patients with influenza. In patients with COVID-19, the frequency of GGO nodules was lower than in patients with influenza, vascular enlargement in GGO was higher, and COVID-19 lesions were more likely to appear in the subpleural, inferior, and posterior lung fields, whereas lung lesions in patients with influenza are more randomly distributed. These differences in chest CT scans may be key in differentiating between the two diseases. What is even more remarkable is that the combined regression model has very good diagnostic performance. 
The genetic overlap between SARS-CoV-2 and SARS-CoV is about 79\%, and that with MERS-CoV is about $50 \%{ }^{14}$. Moreover, SARS-CoV-2 binds to the same cell entry receptor (ACE2) used by SARS-CoV ${ }^{15}$. A recent study analyzed the ACE2 RNA expression profile in the normal human lungs based on the public database and the state-of-the-art single-cell RNA-Seq technique. The result of this study indicated that the ACE2 virus receptor expression is concentrated in a small population of type II alveolar cells (AT2) ${ }^{16}$. Furthermore, this population of ACE2-expressing AT2 also highly expressed many other genes that positively regulating viral reproduction and transmission ${ }^{16}$. The SARS-CoV-2 virus, with a diameter of 60 to $140 \mathrm{~nm}$, can easily reach the periphery of the lung and, as does SARS, bind to ACE2 receptors in the bronchioles, terminal bronchioles, and alveoli. This binding explains why the lesions associated with COVID-19 are mainly distributed in the periphery of the lung at least at the beginning of the infection. Overall, our CT findings and other studies on COVID-19 are similar to those reported for SARS-CoV ${ }^{17-19}$. In contrast, the a2,6-linked sialic acid-bearing receptors, to which human influenza viruses preferentially bind, are abundant in the upper and lower respiratory tract of humans, particularly in tracheobronchial epithelium and type I alveolar cells ${ }^{20}$. Therefore, the influenza pneumonia lesions were not distributed mainly in the peripheral lung but in the central or the whole lung.

According to a brief report on the first systematic autopsy of COVID-19 in China, which has just been prepublished online (Journal of Forensic Medicine, a Chinese journal) ${ }^{21}$, the report revealed significant lung damage, gross bleeding, and large amounts of viscous secretions spilling from the alveoli on cut surfaces. The authors suggest that COVID-19 mainly causes inflammatory responses characterized by deep airway and alveolar injury. This also confirms the previous two case reports on pathology of COVID$19^{22,23}$ and could explain the CT findings of GGO in our study. We can also speculate on the cause of bronchiolectasis within lesions: the extensive alveolar damage, bleeding, and exudation would lead to myxoid material blocking the alveoli, terminal bronchioles, or bronchioles, which would lead to bronchiolectasis in the lesion. This process is rare in cases of influenza pneumonia.

The small vascular enlargement in the GGO is also distinct from that of influenza pneumonia. Neither of the current pathologic studies of COVID-19 mentioned changes about small blood vessel in the lung. However, in the pathological studies of SARS, extensive vascular endothelial injury and damage to epithelial cells were conspicuous features ${ }^{24-26}$.

There are some limitations to our research. Our study was a single-center study. Not all patients were followed to full recovery because some were later referred to infectious disease hospitals in our area. Pediatric, pregnant, and perinatal cases were not included because our hospital does not have a pediatric or an obstetrics and gynecology department. The small sample prevented validating the combined model. Therefore, we cannot be completely confident in the diagnostic performance of this model but hope to include more COVID-19 cases with time. We plan to conduct a prospective, multicenter study, including more cases, to further determine the differences between COVID-19 and influenza pneumonia.

In conclusion, we believe that covid-19 and human influenza pneumonia have certain differences in clinical features and chest CT findings. In clinical practice, the combination of sputum, vascular 
enlargement in GGO and central-peripheral distribution is great helpful for differential diagnosis.

\section{Declarations}

\section{Acknowledgments}

We thank Tom Lang, MA, of Tom Lang Communications and Training International, who edited an early draft of the manuscript. We thank all our colleagues who helped us during the current study.

\section{Contributions}

Z.S., H.Z.X, and B.S made substantial contributions to the conception or design of the work. Z.S. participated in its design, data acquisition and analysis, manuscript preparation; H.Z.X was responsible for design, data analysis, manuscript preparation; Z.H.J., C.Z.X., L.F.M and Z.C.W. were responsible for acquisition and/or interpretation of data. Y.Q. and B.S. were responsible for analysis of data. B.S. put forward the concept of the study, designed the study and coordination, reviewed the manuscript. All authors have read and approved the final version of the manuscript and agree with the order of presentation of the authors.

\section{Competing interests}

The authors declare no competing interests.

\section{References}

1. Thompson WW, Shay DK, Weintraub E, et al. Influenza-associated hospitalizations in the United States. JAMA. 2004. 292(11): 1333-40.

2. Lafond KE, Nair H, Rasooly MH, et al. Global Role and Burden of Influenza in Pediatric Respiratory Hospitalizations, 1982-2012: A Systematic Analysis. PLoS Med. 2016. 13(3): e1001977.

3. Uyeki TM. Influenza. Ann Intern Med. 2017. 167(5): ITC33-ITC48.

4. Shang M, Blanton L, Brammer L, Olsen SJ, Fry AM. Influenza-Associated Pediatric Deaths in the United States, 2010-2016. Pediatrics. 2018. 141(4).

5. Paules C, Subbarao K. Influenza. Lancet 2017;390:697-708.

6. Chen N, Zhou M, Dong X, et al. Epidemiological and clinical characteristics of 99 cases of 2019 novel coronavirus pneumonia in Wuhan, China: a descriptive study. Lancet 2020;395:507-513.

7. Huang C, Wang Y, Li X, et al. Clinical features of patients infected with 2019 novel coronavirus in Wuhan, China. Lancet 2020;395:497-506.

8. Wang M, Zhou Y, Zong Z, et al. A precision medicine approach to managing Wuhan Coronavirus pneumonia. Precision Clinical Medicine 2020.

9. Zou L, Ruan F, Huang M, et al. SARS-CoV-2 Viral Load in Upper Respiratory Specimens of Infected Patients. N Engl J Med 2020. 
10. Holshue ML, DeBolt C, Lindquist S, et al. First Case of 2019 Novel Coronavirus in the United States. N Engl J Med 2020.

11. Yang W, Sirajuddin A, Zhang X, et al. The role of imaging in 2019 novel coronavirus pneumonia (COVID-19). Eur Radiol 2020.

12. Huang, Z. et al. Imaging features and mechanisms of novel coronavirus pneumonia (COVID-19): Study Protocol Clinical Trial (SPIRIT Compliant). Medicine (Baltimore) 99, e19900 (2020).

13. Li Q, Guan X, Wu P, et al. Early Transmission Dynamics in Wuhan, China, of Novel CoronavirusInfected Pneumonia. N Engl J Med 2020.

14. Lu R, Zhao X, Li J, et al. Genomic characterisation and epidemiology of 2019 novel coronavirus: implications for virus origins and receptor binding. Lancet 2020;395:565-574.

15. Zhou $P$, Yang $X L$, Wang $X G$, et al. A pneumonia outbreak associated with a new coronavirus of probable bat origin. Nature 2020.

16. Zhao Y, Zhao Z, Wang Y, Zhou Y, Ma Y, Zuo W. Single-cell RNA expression profiling of ACE2, the putative receptor of Wuhan 2019-nCov. bioRxiv 2020:2020.01.26.919985.

17. Chan MS, Chan IY, Fung KH, Poon E, Yam LY, Lau KY. High-resolution CT findings in patients with severe acute respiratory syndrome: a pattern-based approach. AJR Am J Roentgenol 2004;182:49-56.

18. Hui JY, Cho DH, Yang MK, et al. Severe acute respiratory syndrome: spectrum of high-resolution CT findings and temporal progression of the disease. AJR Am J Roentgenol 2003;181:1525-38.

19. Ooi GC, Khong PL, Müller NL, et al. Severe acute respiratory syndrome: temporal lung changes at thin-section CT in 30 patients. Radiology 2004;230:836-44.

20. Rogers GN, Paulson JC. Receptor determinants of human and animal influenza virus isolates: differences in receptor specificity of the $\mathrm{H} 3$ hemagglutinin based on species of origin. Virology 1983;127:361-73.

21. Liu Q, Wang RS, Qu GQ et al. Gross examination report of a COVID-19 death autopsy. 36(1),2020.

22. Xu Z, Shi L, Wang Y, et al. Pathological findings of COVID-19 associated with acute respiratory distress syndrome. Lancet Respir Med 2020.

23. Tian S, Hu W, Niu L, Liu H, Xu H, Xiao S. Pulmonary Pathology of Early Phase SARS-COV-2 Pneumonia .

24. Ding $Y$, Wang $H$, Shen $H$, et al. The clinical pathology of severe acute respiratory syndrome (SARS): a report from China. J Pathol 2003;200:282-9.

25. Gu J, Gong E, Zhang B, et al. Multiple organ infection and the pathogenesis of SARS. J Exp Med 2005;202:415-24.

26. Hwang DM, Chamberlain DW, Poutanen SM, Low DE, Asa SL, Butany J. Pulmonary pathology of severe acute respiratory syndrome in Toronto. Mod Pathol 2005;18:1-10.

\section{Figures}




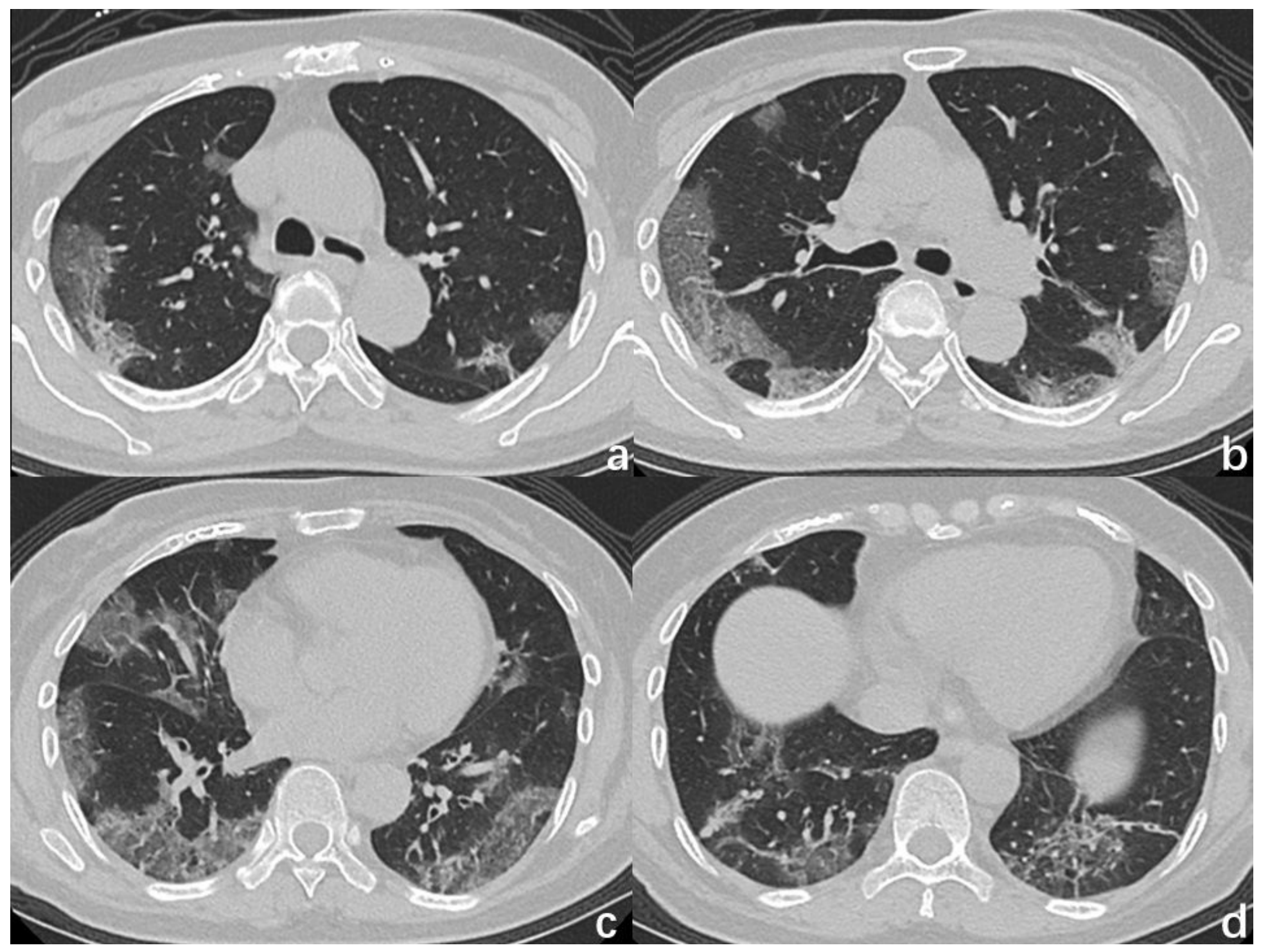

Figure 1

A 66-year-old man with COVID-19 infection, presenting fever with cough for 7 days. CT images showed multiple GGOs in bilateral lung, mainly distributedin the subpleural areas, with reticular pattern (interlobular septal thickening). 


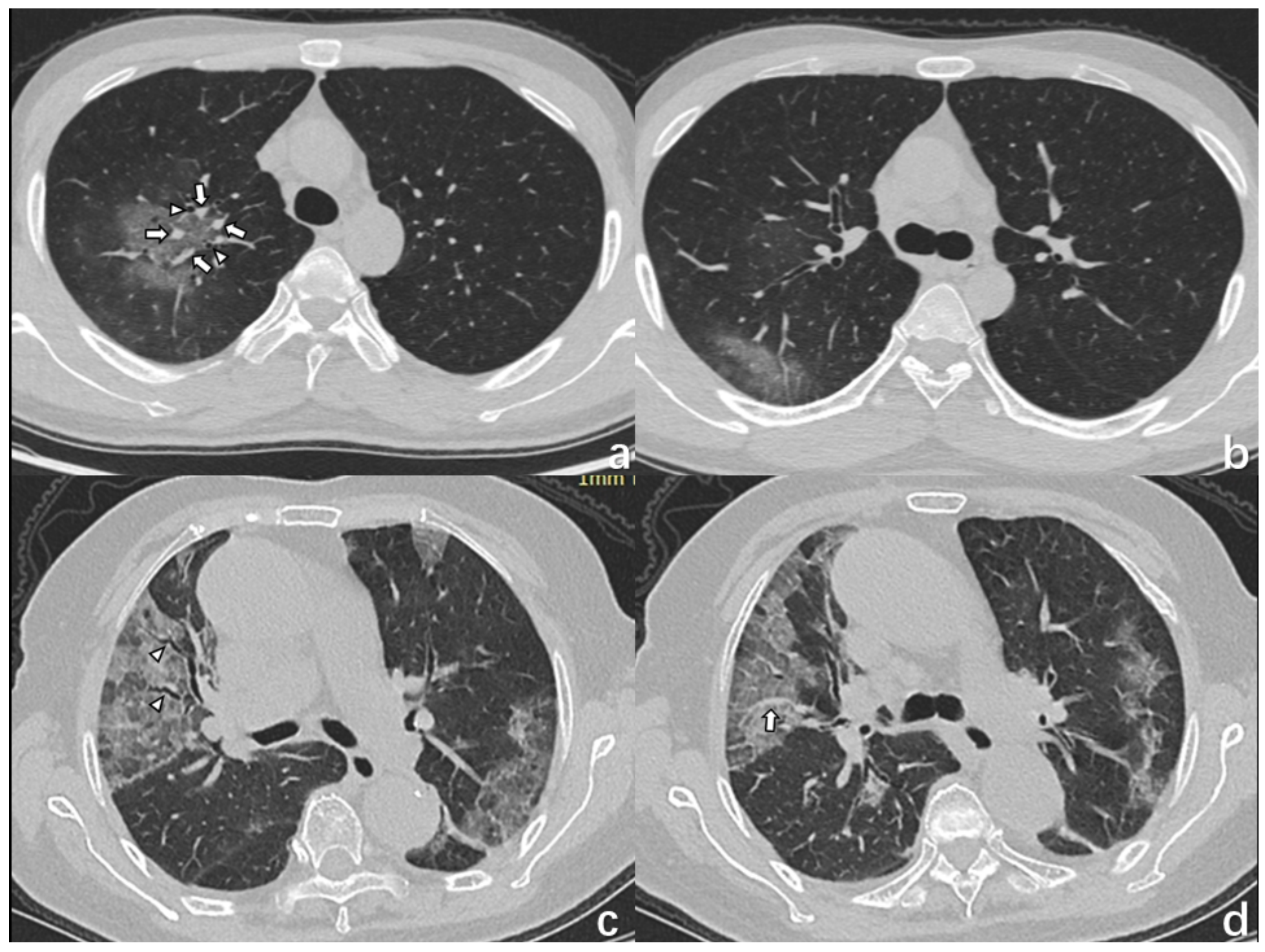

Figure 2

a b A 33-year old woman with COVID-19 infection, living in Wuhan presented with fever, rhinocleisis, and dry cough. Axial (a) and coronal (b) CT images show a subpleural mixed ground-glass opacity with multiple bronchiolectasis (triangles) and small vascular enlargement (arrow) in the right lower lobe. $\mathrm{c} \sim \mathrm{d}$ A 47-year old man with a COVID-19 infection, who traveled to Wuhan presented with fever. Axial (c) and coronal (d) CT images showed dominantly posterior- and inferior, multiple, GGOs with bronchiolectasis (triangles) and vascular enlargement (arrows). 


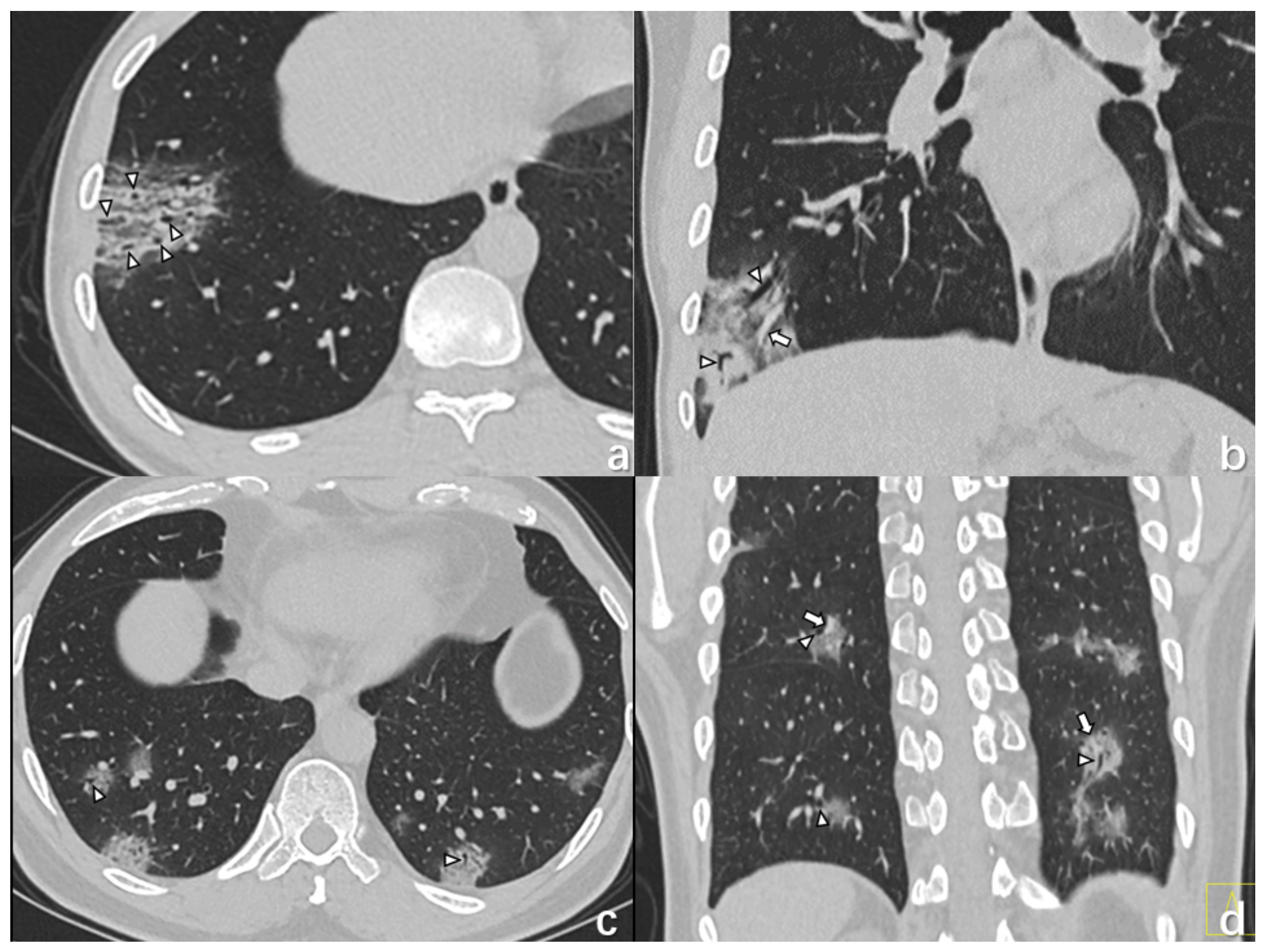

Figure 3

a b A 35-year-old male with COVID-19 infection, presenting fever and headache for 1 day. CT images showed small vascular enlargement (arrows) and bronchiolectasis (triangles) in GGO, as well as multiple GGOs in the subpleural areas of bilateral lung. c d An 80-year old man with COVID-19 infection, who was lived in Wuhan presented with fever and dry cough. CT images showed multiple patchy areas of GGOs as crazy-paving pattern with bronchiolectasis (triangles) and vascular enlargement (arrows). 


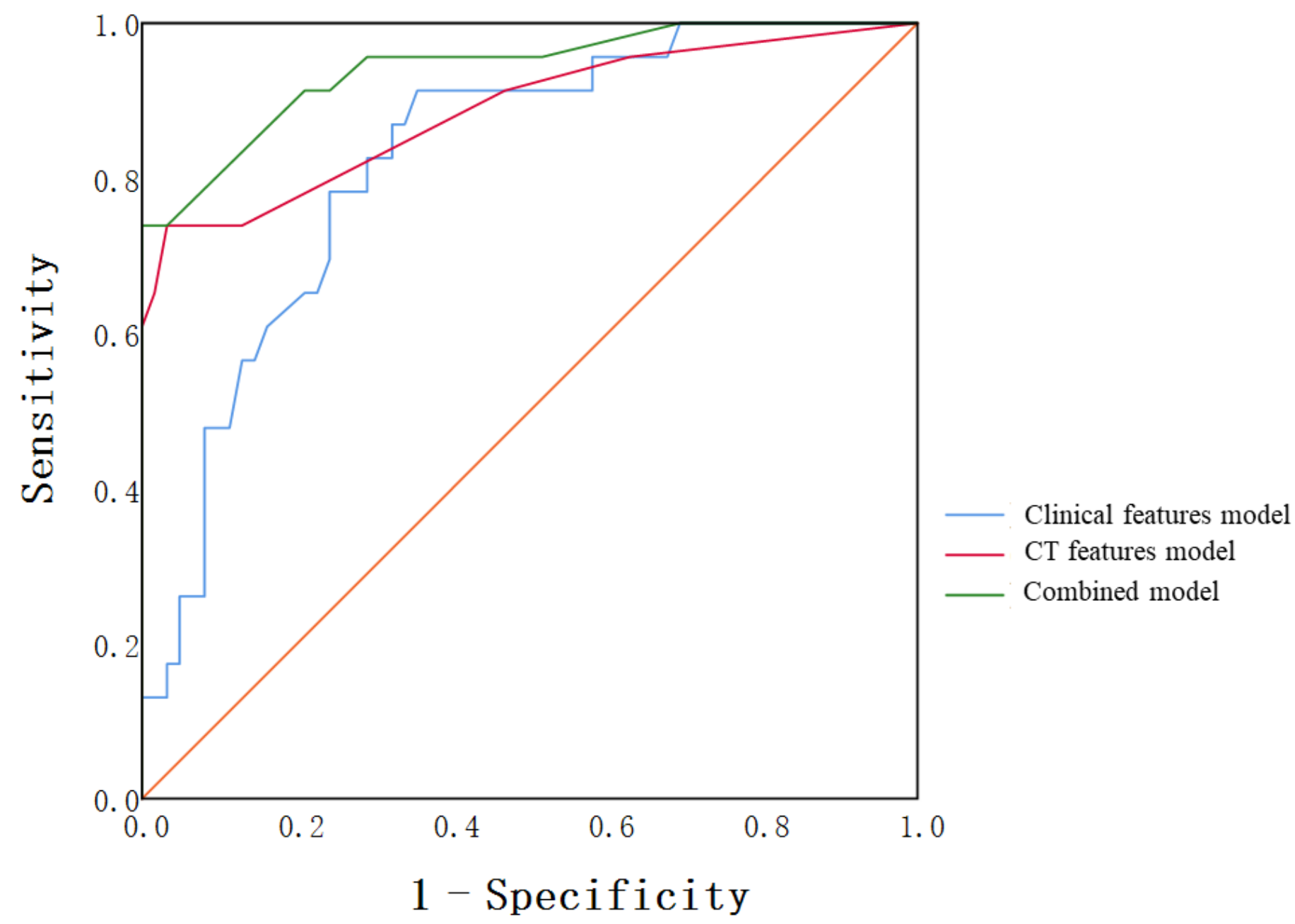

Figure 4

The performance characteristics of the logistic regression models for diagnosing patients with COVID-19 infection. Blue line, the model based on clinical factors; red line, the model based on CT features; green line, the combined model with both clinical and CT features. 